\title{
Los Determinantes Sociales de Salud y la lucha por la equidad en Salud: desafíos para el Estado y la sociedad civil
}

\section{The Social Determinants of Health and the Struggle for Health Equity: challenges for States and civil society}

\section{Eugenio Villar}

Medico salubrista peruano, Maestría en salud Comunitaria (Londres). Departamento de Equidad, Pobreza y Determinantes Sociales de la Salud de la OMS. Ginebra, Suiza.

Endereço: 20, Avenue Appia, $\mathrm{CH}-1211$ Geneva 27, Suiça.

E-mail: villareळwho.int

\section{Resumen}

La OMS ha creado una Comisión de los Determinantes Sociales de la Salud que producirá su reporte en el 2008. Aunque el tema de los determinantes sociales (DSS) de la salud no es nuevo, la nueva visibilidad global en el tema refleja el agotamiento del modelo de desarrollo neoliberal que ha agudizado la inequidad y consecuentemente hace resurgir el tema de la justicia social. Nuevos enfoques y evidencia ubican la inequidad en salud como resultado de la inequitativa distribución de los DSS. Procesos en curso como la globalización de signo neoliberal y el cambio climático agudizan las inequidades así como la exclusión en salud al actuar directa y desigualmente sobre los DSS. Algunos ejemplos de políticas publicas exitosas: Suecia, dado su particular contexto político-institucional ha desarrollado una política (intersectorial) de salud publica cuyo objetivo es disminuir la inequidad diseñando estrategias participativas sobre sus DSS. Chile viene también construyendo inclusivamente un sistema de protección social infantil (Chile Crece Contigo) a partir de una integración de programas bajo un norte de derechos y de DSS. La meta es la cobertura universal con un enfoque de ciclo de vida. Finalmente, la Sociedad Civil de las Américas viene desarrollando un debate hacia una agenda común en el tema de los DSS, con los gobiernos de Brasil y Chile así como la OEA, OPS y OMS. Los ejes son la lucha por un nuevo modelo de desarrollo participativo y sostenible que democratice el abordaje de los DSS bajo un enfoque de derechos.

Palabras-clave: Determinantes Sociales; Justicia Social; Políticas Públicas; Desenvolvimiento Participativo. 


\section{Abstract}

WHO has launched the Commission on Social determinants of Health which will deliver its final report in 2008. The social determinants of health (SDH) approach is not a new subject but one which has gained more visibility with the widening of inequities, blamed on the neo-liberal development model. Progressively, social justice issues are re-emerging, backed by evidence and new approaches which crystallize the relationship between unequal distribution of SDH and their consequences, unequal health outcomes.

Processes like globalization under a neo-liberal brand and climate change influence the SDH, resulting in acute inequities and social marginalization. There are some successful examples of countries with policies addressing the SDH. For example, Sweden, with due regard to its particular political and institutional context, has developed public health intersectoral policies that aim at decreasing health inequities. These policies also encourage a participatory model as a means of efficiently tackling the SDH. Another example is Chile which is building a children social protection system (Chile Crece Contigo) using an integrated approach that includes right-based and SDH approaches. The programme's objective is universal coverage with a life cycle approach. A major milestone for civil society in Latin America is the effort by the Americas' Civil Society organizations to strengthen a dialogue that fosters a common agenda on the SDH. The organizations are working with the governments of Brazil, Chile, the OIAS, PAHO and WHO. Their goal is to introduce a new participatory and sustainable development mode, one which is participatory, rightsbased and ensures sustainable improvements of SDHs and benefits all people.

Keywords: Social Determinants; Social Justice; Public Policies; Participatory Development.

\section{Introducción}

El tema de los Determinantes Sociales de la Salud (DSS) evidentemente no es nuevo y menos aun en nuestra Región. Referido genéricamente por la Comisión de Determinantes Sociales de la Salud de la OMS (CDSS) como "el conjunto de las condiciones sociales en las cuales la gente vive y trabaja” (OMS, 2007), éstos han sido hechos explícitos y han sido atendidos en la historia de la humanidad desde la antigüedad por diversas culturas. En muchas de ellas los DSS se han mantenido como un elemento central de su ideología y vida cotidiana hasta el presente, como por ejemplo en las poblaciones indígenas. En ellas la salud es concebida holisticamente con la tierra, la naturaleza y la vida social y espiritual. Los DSS estuvieron posteriormente a la base de los fundadores de la salud pública moderna. Ha sido sin duda Rudolf Virchow (18211902) quien analizo y escribió en su tiempo sobretodo la determinación social de la enfermedad y su relación también con la política (Virchow, 1985).

Mas recientemente, la Constitución de la OMS, en $1946^{1}$, reflejando lo más avanzado del pensamiento medico social que estuvo muy presente desde la sección de salud de la Liga de Las Naciones (que precedió a la creación de las NNUU), es la que consagro la definición de la salud como "un estado de pleno bienestar físico mental y social y no solo la ausencia de enfermedad" así como un derecho fundamental de los individuos (antecediéndose incluso en ese aspecto a la Declaración Universal de los Derechos Humanos). Dicha Constitución también hizo explicito dentro de las funciones de la OMS la colaboración con los estados miembros y los organismos especializados apropiados "para promover el mejoramiento de la nutrición, la habitación, el saneamiento, la recreación, las condiciones económicas y de trabajo y otros aspectos de la higiene del medio". En la segunda mitad del siglo pasado la Conferencia Internacional de Alma Ata en 1978 (OMS, 1978) consideró también como uno de los pilares básicos de la estrategia de la Atención Primaria de la Salud a los DSS declarando "La necesidad de una estrategia integral de salud que no solo preste servicios de salud sino que también aborde las causas sociales, económicas y políticas subyacentes de la mala salud". 
Vale entones preguntarse por que es que habiendo enfoque holístico de los DSS desde hace tanto tiempo y habiendo sido su abordaje desde las políticas, materia de consensos globales, por que es que lejos de materializarse éstos mas bien han sido negligidos tanto por las políticas de salud como por las de desarrollo, en la mayoría de los países? Que implicancias ha tenido esta negligencia? Y sobre todo que es necesario y que es posible hacer a partir de la evidencia y cuales son los actores llamados a la acción? Estas son algunas de las preguntas que pretendemos discutir a fin de fomentar el debate.

\section{Elementos Conceptuales e Históricos}

Irwin y Solar ${ }^{2}$ proponen a la CDSS un marco conceptual a partir de un enfoque holístico que da cuenta de la evolución histórica de los valores y la ideología así como de la evidencia científica interdisciplinaria en estos temas. A partir de ello queda clara la existencia de una serie de dimensiones interrelacionadas que parten del contexto estructural socio económico y político (distal al resultado final de salud y bienestar) de la sociedad. Dicho contexto se traduce en una estratificación de la población en clases sociales en términos de la distribución del excedente económico así como del poder en sus diferentes esferas. Esta estratificación social - mediada por el grado de cohesión o capital social- da paso a lo que serian los factores intermediarios (DSS) expresados en una (desigual) distribución de 3 tipos de factores.

Estos factores determinan patrones de exposición y de vulnerabilidad diferenciados. Tendríamos entonces por un lado los factores de vivencias materiales en las áreas de vivienda, trabajo, alimentación, saneamiento etc.; por otro los factores psicosociales y conductuales; y por ultimo ciertamente los factores biológicos (que incluyen los genéticos). Un determinante importante en esta esfera intermedia serian los mismos sistemas de salud, los cuales, en este enfoque, devendrían en determinantes sociales en la medida que son el resultado de construcciones sociales muy vinculado a las políticas. La interacción de estos DSS influiría (próximamente) a explicar la salud y enfermedad de los individuos.

En la esfera de las respuestas congruentes con este enfoque holístico los mismos autores proponen 4 tipos de políticas publicas (cada una con posibles expresiones en niveles de lo global a lo local). Las que abordan (distalmente) a la estratificación social a fin de disminuir las inequidades mitigando los efectos de dicha estratificación, las diseñadas a nivel intermedio para reducir la exposición o la vulnerabilidad de las poblaciones más desfavorecidas y por ultimo las políticas que actúan (proximalmente) reduciendo las consecuencias desiguales en el estado de salud o su compensación en la esfera social o económica. Dichas políticas serian notablemente influidas por los enfoques intersectoriales y por el nivel de participación social empoderante.

Estos marcos conceptuales nos ayudarían a explicar el por que los grandes intereses económicos y consecuentemente políticos surgidos a partir del desarrollo del complejo medico industrial y del mercado privado de la salud favorecieron el desarrollo de tecnologías diagnosticas y de tratamiento (proximal y recuperativo) de la salud en las cuales la acumulación de excedente es mas significativo. Esto se hizo en desmedro de medidas estructurales y del desarrollo de políticas públicas que abordaran más integralmente los DSS. Por otro lado existe creciente evidencia que demuestra no solo el aumento significativo de la inequidad en salud (medible entre los países y a su interior) sino que ese incremento responde también al incremento en la inequidad en los DSS, en particular el ingreso, y no solo al acceso o no a los servicios de salud. Es por ello que el abordaje de los DSS, en el enfoque de la CDSS, se considera contribuiría sustantiva e integralmente al abatimiento de la inequidad en salud ${ }^{3}$.

El contexto de la guerra fría no habría sido tampoco conducente a los abordajes integrales de la problemática social por los alineamientos de los países con una de las superpotencias, lo cual resto en casi todos los casos protagonismo nacional para el desarrollo y más bien acentuó la dependencia a ejes geopolíticos ajenos.

\footnotetext{
2 WHO. Commission on Social Determinants of Health. A conceptual framework for action on the social determinants of health. Geneva, 2006. Discussion paper by Alec Irwin and Orielle Solar.

3 WHO. Basic documents. Geneva, 2005.
} 
Esto ocurrió apesar del hito que constituyo la creación de las NNUU y de la OMS y la aprobación de la declaración Universal de los DDHH hacia mediados del siglo XX. La Conferencia de Alma Ata que se enmarco en la apertura de un espacio caracterizado por la fortaleza de los No Alineado y el grupo de los 77 y por el planteamiento del Nuevo Orden Económico Mundial, hubiera ofrecido un marco propicio para el desarrollo de los DSS dentro de paradigmas de desarrollo con mayor énfasis en lo social.

Lamentablemente dicha coyuntura fue corta y fue también seguida, luego de la crisis de la deuda, por los programas de ajuste estructural, el Consenso de Washington y el colapso de la Unión Soviética que dio fin a la guerra fría. Todo ello favoreció el desarrollo del neoliberalismo y la globalización bajo ese signo. Este marco internacional, ajeno a las perspectivas holísticas de ataque a las causas de la mala salud, sin duda facilito el desarrollo de enfoques verticales o de APS selectiva, que en esencia se alejan del enfoque de DSS. Vale la pena enfatizar que siendo importantes estas evoluciones geopolíticas algunos países o regiones dentro de ellas pudieron desarrollar experiencias importantes en base a sus desarrollos políticos locales. Es el caso de países como Cuba y Costa Rica en nuestra región.

La coyuntura mundial actual estaría marcada por el inicio de un desgaste del modelo neoliberal a partir de las tensiones generadas por la creciente inequidad como resultado de una obsesión por el crecimiento económico con poca generación de empleo digno y una negligencia o incapacidad del rol redistributivo del Estado. A todo ello se suma una creciente preocupación por el cambio climático, el cual viene ya afectando a un sinnúmero de poblaciones marginadas y se constituye como una posible amenaza para toda la humanidad. Este proceso, sin ser social sino ambiental en primera instancia, se ha agudizado a partir del modelo de desarrollo y de globalización en curso y se expresa en inequidades acentuadas ya que son y serán los más pobres los que padezcan más sus consecuencias a menos que se tomen acciones para contrarrestarlo y atenuar sus efectos. En otras palabras, el cambio climático agudizaría la inequidad en salud a través del deterioro de los DSS especialmente de los más pobres y marginados.

En la coyuntura actual resulta ilustrativa la creciente atención que se viene brindando al tema de la equidad concebida en la práctica no con un enfoque de derechos sino únicamente como instrumental para el desarrollo sostenible y la gobernabilidad, la cual se ve peligrar. Relacionado a esto último se observa que nuestra Región latinoamericana, que es la que exhibe mayores índices de inequidad en el planeta, sea la que viene presentando profundos cambios políticos que vienen poniendo el tema de la pobreza e inequidad en el centro de la agenda política. A nivel global ello viene conduciendo a una mayor atención al tema de la justicia social y con el a los DSS. Es en ese marco que la OMS lanza la CDSS en el 2005, cuyo Reporte será hecho publico a inicios del 2008 propiciamente coincidente con la celebración de los 6 o años de creación de la OMS y los 30 de la realización de la Conferencia de Alma Ata (Lee, 2003).

\section{Que Hacer?: el desafío de las políticas públicas y la movilización social}

En función del marco político-institucional, el abordaje de los DSS se constituye un reto para los Estados en los siguientes términos:

1. Como definir inclusivamente las políticas de desarrollo en un marco de derechos a fin de abordar el problema estructural de la inequidad a través de políticas económicas, sociales y medio ambientales que aseguren generación y distribución equitativa y sostenible de riqueza y que aborden los DSS y no solo sus efectos? Como construir ciudadanía y democracia a través de reformas políticas? Coherente con lo anterior, como replantear políticamente a nivel internacional el proceso de globalización en curso como generador de inequidad?

2. Como avanzar desde la evidencia y el discurso explicativo hacia la formulación y desarrollo de políticas intersectoriales? Como lograr que éstas aborden de una manera más comprehensiva los DSS en el marco de los nuevos modelos de desarrollo ya mencionados? 3. En el marco de lo anterior, que rol le compete específicamente al sector salud? Como desarrollar el liderazgo y la capacidad institucional del sector, incluyendo la coordinación intersectorial, a fin de abordar los DSS hacia una mejor equidad en salud? Como desarrollar sistemas de salud de acceso universal y que vía la promoción de la salud se articulen con acciones de 
otros sectores a fin al menos de atenuar las causas de la inequidad en salud?

4. Como desarrollar las instituciones del sector para la apertura de mecanismos que fomenten la participación social en el todo el desarrollo de las políticas especialmente en la rendición de cuentas? Asimismo, como se articulan estos nuevos mecanismo de democracia participativa con la democracia representativa sea esta local o nacional?

5. Como se generan consensos y alianzas políticas que aseguren liderazgo para el cambio y sostenibilidad política que incluya la imprescindible ampliación del espacio fiscal para la salud, sin que ellos sea visto como una amenaza para la estabilidad macroeconómica y la necesaria competitividad del país?

Desde el lado de la sociedad civil los retos no son menos importantes:

1. Como educar, movilizar, organizar y empoderar a la población, especialmente la más marginada (y con menos cohesión/capital social) a fin de asegurar la participación efectiva en la exigencia, la formulación, el cabildeo, la implementación, el monitoreo y la rendición de cuentas en los niveles locales, intermedios, nacionales y globales?

2. Como hacer el mejor uso del enfoque de derechos para la exigibilidad política y jurídica a los estados, haciendo uso del marco del derecho a la salud de manera indivisible con el conjunto de los derechos humanos? 3. Como construir la democracia participativa articulada con una democracia representativa más legitimada a partir de políticas publicas inclusivas, desarrollo de capacidades institucionales para el cambio y el escrutinio y vigilancia de los presupuestos públicos? 4. Como evitar la burocratización, cooptación y corrupción de los liderazgos y los movimientos sociales especialmente por parte de gobiernos populistas?

\section{Experiencias Innovadoras de Políticas y de Movilización Social}

\section{Suecia}

Referimos resumidamente el ejemplo de Suecia el cual teniendo un contexto político, cultural, institucional y económico muy diferente al latinoamericano se lo puede darnos algunas ideas.
A inicios de la década Suecia inicio un proceso innovador en el desarrollo de sus políticas de salud pública a partir de un enfoque de determinantes sociales de la salud. En cuanto a su formulación el país definió unos pocos objetivos a alcanzar estructurándolos de manera bastante sencilla, a diferencia de otros países.

El aspecto central fue que asumiendo como meta la disminución de las inequidades en salud (en un país con una cultura de igualdad social y con gran cohesión social), Suecia desarrollo evidencia de cuales eran las causas del incremento de la inequidad en salud y a continuación baso sus políticas en el abordaje de dichas causas.

Dos elementos a destacar de dicho proceso:

1. En términos de los contenidos de las políticas sus estrategias están basadas en el abordaje de los DSS y necesariamente con un enfoque intersectorial. Esto se aleja de los enfoques tradicionales epidemiológicos que centran el accionar de sus políticas en el abordaje de las consecuencias del proceso salud enfermedad, vale decir en la mala salud.

2. En términos de los procesos, Suecia baso toda la discusión y definición de sus políticas asegurando una amplia participación social, vale decir incluyendo todos los actores políticos relevantes. El objetivo era alcanzar consenso social, el cual fue subsecuentemente cristalizado en el Plan de Salud del gobierno.

Se definieron en consecuencia 6 áreas de acción, por cierto especificas para su contexto:

1. Incrementar la cohesión/capital social incluyendo reducciones en la discriminación contra minorías así como la promoción de la democracia local

2. Promover mejores condiciones de trabajo a través de la disminución del estrés crónico, la promoción de la participación de los empleados en su ambiente laboral así como la promoción de esquemas flexibles de trabajo

3. Mejoría de las condiciones para niños y jóvenes a través de mecanismos universales de apoyo social para las familias con niños pequeños y escuelas promotoras de la salud

4. Mejoría del ambiente físico a través de la coordinación de intervenciones medio ambientales y de salud 5. Promoción de estilos de vida saludables 
6. Apoyo a la educación e investigación en salud publica a fin de asegurar una adecuada evidencia para el desarrollo de las políticas e intervenciones

\section{Chile}

Referimos a continuación otro ejemplo en construcción, esta vez en nuestra región. Se trata del Sistema "Chile Crece Contigo". Dicho sistema se basa explícitamente en un enfoque de derechos y de DSS articulado desde la oferta de servicios pero fomentando una amplia participación incluso de los mismo niños desde su diseño. El sistema esta a cargo directamente del Gabinete Social que incluye a los ministros de dichas carteras y que reportan a la Presidente del país en su desarrollo.

Este se inscribe en una lógica modular que va incorporando progresivamente varios programas nuevos y existentes (anteriormente dispersos y algunos yuxtapuestos) a fin de construir un Sistema de Protección Social progresivo y universal, es decir accesible a todos los ciudadanos sin excepción. En ese sentido es un eslabón articulado al Plan Auge (prestaciones mininas garantizado por el Estado radas en los servicios de salud), Chile solidario (que incluye transferencias de efectivo condicionadas pero articuladas con varias otras formas de asistencia social), la seguridad social contributiva así como las políticas de subsidios públicos inscritas en la lucha contra la pobreza y la exclusión social del país.

La estrategia de intervención consiste en encadenar acciones de apoyo a servicios sociales con un enfoque de ciclo de vida que considera a la infancia como sujeto final de la intervención y la participación de la familia (también los niños), la comunidad y la institucionalidad social. Las dimensiones de intervención se dan a partir de un abordaje simultáneo de distintas áreas de la vida del niño y su familia entendiendo que cada una constituye un aspecto fundamental, insustituible e impostergable para el adecuado desarrollo infantil. Por el momento este abarca las siguientes áreas:

1. Identificación/registro civil

2. Salud

3. Aprendizaje

4. Entorno familiar

5. Habitabilidad
6. Ingresos y trabajos familiares

7. Cuidado Infantil para que las madres trabajen fuera del hogar.

En el caso de la salud específicamente este incluye, con un enfoque de ciclo vital, el control de la gestación, el nacimiento, la alimentación de la madre y del niño, la prevención y el control de las enfermedades, la detección precoz y el abordaje de la discapacidad, la salud mental y el control del consumo de sustancias adictivas.

\section{Movilización de la sociedad civil regional}

Finalmente queremos referir una experiencia Regional inicial y reciente promovida por los gobiernos de Brasil y de Chile, por organismos de la sociedad civil latinoamericana y por la OEA y la OPS/OMS en el marco del trabajo de la CDSS. Se trato de una Consulta con un grupo ampliado de la sociedad civil latinoamericana a fin de debatir sobre los DSS y las perspectivas para la acción en la región y dando aportes a nivel global, incluyendo al reporte de la CDSS que será presentado en Mayo del 2008.

El proceso fue liderado por el Departamento de Apoyo a la Gestión Participativa del Ministerio de Salud Publica del Brasil a partir de la trayectoria y liderazgo que Brasil tiene en el tema de los DSS. Este se expresa en la conformación de una Comisión Nacional de DSS (único país en el mundo que cuenta con una comisión ya operativa). La iniciativa de la consulta genero adhesiones por parte del Gobierno de Chile, que como ya se refirió viene impulsando el enfoque de DSS y de la Secretaria de Cumbres de las Américas de la OEA así como de la OPS y la OMS (esta ultima actúa como secretariado de la CDSS global).

Es de resaltar que la OPS/OMS debatió a nivel de la Reunión Anual de Ministros de Salud en Septiembre del 2006 el tema de los DSS dada la creciente preocupación por el incremento de la inequidad en salud y la insuficiencia de abordajes a la misma que vayan más allá de la recuperación curativa de la salud. La consulta, realizada en Brasilia en Abril del 2007, congrego alrededor de 40 organizaciones de la sociedad civil de la Región incluyendo representantes de mujeres, campesino/as, pueblos de la selva, pueblos y nacionalidad indígenas, comunidades de afro descendientes, pueblos gitanos y otros pueblos nómadas, de orientación e identidad de genero (LGTB), de organizaciones 
territoriales y vecinales, sindicatos de trabajadores/ as, académicos, viviendistas, usuarios de servicios de salud y grupos de enfermos, gremios profesionales y de ONGs.

Los puntos más resaltantes acordados por la Consulta fueron los siguientes ${ }^{4}$ :

1. El enfoque de DSS genera necesariamente una critica profunda al modelo de desarrollo neoliberal y al proceso de globalización con dicho signo. A ellos se les percibe como generadores de inequidad en salud a partir de la mercantilización y privatización de la salud, de su papel en la reducción del rol del estado como promotor de la salud y garante del derecho humano a la salud y de la concentración para unos pocos y la marginación a poblaciones enteras en el goce de los DSS. En consecuencia el abordaje de los DSS pasa por la superación de dicho modelo hacia un nuevo modelo de desarrollo económico y social sustentable.

2. La reafirmación que la salud es un DERECHO HUMANO garantizado por el Estado y no una mercancía sujeta a las reglas del mercado. El derecho a la salud implica derechos tanto en el acceso universal a los servicios de salud y educación (superando la marginación a la que actualmente son sujetos muchos de los grupos representados en la consulta); como también derechos en los determinantes sociales como vivienda, alimentación, trabajo digno, participación ciudadana, entre otros. Además se enfatiza que tanto los derechos humanos y los DSS son indivisibles como interdependientes.

3. Las organizaciones se plantean en consecuencia una agenda común en el tema de los DSS a fin de fortalecer el liderazgo y protagonismo de la sociedad civil a fin de participar con los estados en el desarrollo de políticas publicas integrales que aborden los DSS. En ese sentido se plantean alianzas entre la sociedad civil las cuales compartan las experiencias a fin de fortalecer la acción transformadora de los DSS y la exigibilidad de la garantía del derecho a la salud.

4. Finalmente se hace un llamado a los gobiernos y organizaciones internacionales a fin de que continúen dando impulso al proceso iniciado con la creación de la CDSS asegurando una amplia e independiente participación de la sociedad civil en dicho proceso.

\section{Referências}

LEE, J. W. Global health improvement and WHO: shaping the future. The Lancet, London, v. 362, p. 2083-2088, 2003.

OMS - ORGANIZACIÓN MUNDIAL DE LA SALUD. Conferencia Internacional sobre Atención Primaria de Salud. Declaración de Alma Ata. Alma-Ata, 1978. Disponível em: <http://www.paho.org/Spanish/dd/ pin/alma-ata_declaracion.htm $>$. Acesso em: 03 abril de 2007.

OMS - ORGANIZACIÓN MUNDIAL DE LA SALUD. Comisión sobre Determinantes Sociales de la Salud. Acción sobre los factores sociales determinantes de la salud: aprender de las experiencias anteriores. Geneva, 2005. Disponível em: <http://www.who.int/ social_determinants/resources/action_sp.pdf $>$. Acesso em: 01 abril de 2007.

OMS - ORGANIZACIÓN MUNDIAL DE LA SALUD. La Comisión de determinantes sociales de la salud. Geneva, 2007.

VIRCHOW, R. Collected essays on public health and epidemiology. Cambridge: Science History

Publications, 1985. 